$\Rightarrow$ MARKET WATCH

\title{
Upcoming market catalysts in Q3 2019
}

Potential market catalysts in the third quarter of 2019 include top-line clinical trial results for AP-CD/LD (accordion pill carbidopa/levodopa; developed by Intec Pharma) for Parkinson disease, as well as US approval decisions for two first-in-class therapies: lumateperone (developed by Intra-Cellular Therapies) for schizophrenia and golodirsen (developed by Sarepta Therapeutics) for patients with Duchenne muscular dystrophy (DMD) with a confirmed mutation in the dystrophin gene that is amenable to skipping exon 53.

Results from the phase III ACCORDANCE trial evaluating AP-CD/LD, Intec's novel $\mathrm{CD} / \mathrm{LD}$ pill utilizing both immediate and controlled-release technology for the treatment of Parkinson disease, are expected in July or August. These will be the first phase III data available for the drug, which has performed well in phase II. Top-line results from IN18001, a phase II trial comparing AP-CD/LD with Sinemet, an FDA-approved immediaterelease formulation of $\mathrm{CD} / \mathrm{LD}$, showed a

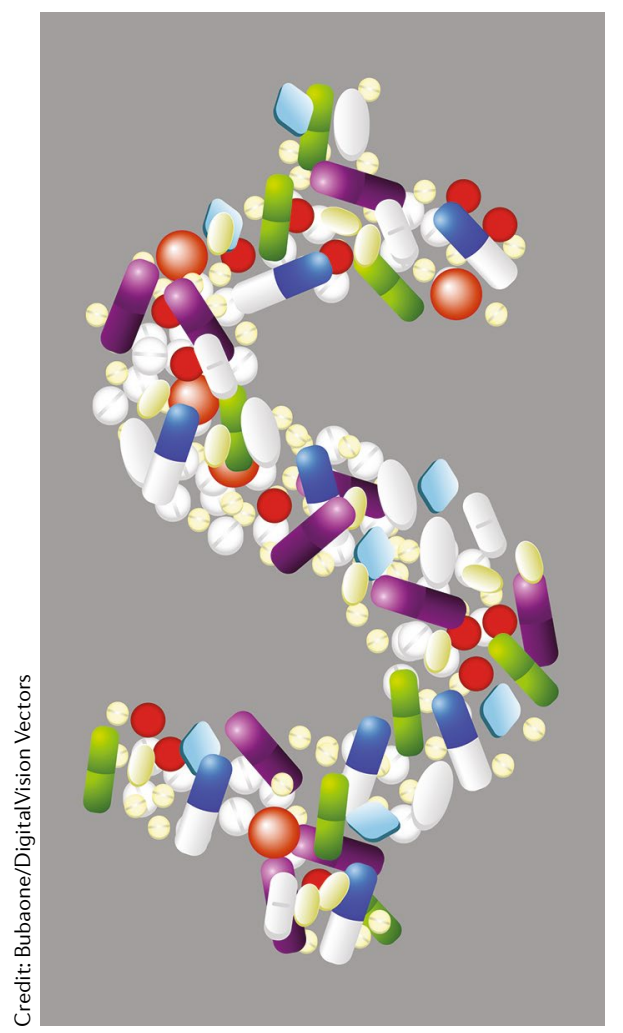

mean difference of 0.63 points $(P<0.047)$ in the levodopa fluctuation index in favour of AP-CD/LD. Intec's formulation of $\mathrm{CD} / \mathrm{LD}$ is also more convenient; patients only take AP-CD/LD two or three times daily, depending on their disease progression, whereas current standard-of-care calls for at least four doses of CD/LD daily. The primary end point of the placebo and comparatorcontrolled phase III ACCORDANCE trial is change in baseline of daily 'off time', that is, the time in which patients suffer symptomatic episodes typical to Parkinson disease, such as difficulty walking and problems with coordination. Favourable phase III data compared with Sinemet could bolster the likelihood of approval for $\mathrm{AP}-\mathrm{CD} / \mathrm{LD}$, which is poised to go through the simpler 505(b)(2) pathway to FDA approval.

Lumateperone, a small-molecule modulator of serotonin, dopamine and glutamate signalling, is under FDA review for the treatment of schizophrenia, with a Prescription Drug User Fee Act (PDUFA) action date of 27 September. Lumateperone acts as both a presynaptic partial agonist and postsynaptic antagonist at $\mathrm{D}_{2}$ receptors, and has also been observed to have potent interactions with $5-\mathrm{HT}_{2 \mathrm{~A}}$ receptors, $\mathrm{D}_{1}$ receptors with indirect glutamatergic modulation and serotonin transporters. Its unique mechanism of action has the potential to contribute to a broader range of efficacy against disease symptoms than currently available antipsychotics. Furthermore, lumateperone's favourable safety profile in initial clinical trials contributed to its fast track designation by the FDA, although this has been overshadowed by long-term safety issues in non-human toxicology studies that only surfaced when the drug was in phase III development. Initial results from one of the three randomized, double-blind, placebocontrolled trials in the phase III programme, study 302 , showed that lumateperone failed to differentiate from placebo on the primary end point, positive and negative syndrome scale (PANSS) score. However, statistically significant separation from placebo on this primary end point was shown in the other two trials. Approval of lumateperone could be a disruptive force to the market for medications for schizophrenia owing to its potential for an improved safety profile over current first-line antipsychotics. Lumateperone could also be Intra-Cellular's first marketed drug.

DMD is an $\mathrm{X}$-linked recessive disorder characterized by progressive muscle degeneration and weakness owing to mutations that affect the function of dystrophin, a protein involved in maintaining the integrity of muscle fibres. Golodirsen is a form of antisense therapy that skips a mutated exon in the dystrophin gene (specifically, exon 53), with the aim of producing more functional dystrophin protein. The only available clinical trial data for golodirsen comes from an analysis of 25 of the patients that received golodirsen in a phase I/II study in Europe. The results revealed only modest, yet statistically significant, increases from baseline to week 48 in mean dystrophin production, from $0.095 \%$ to $1.019 \%(P<0.001)$. These are similar to the increases seen in the clinical trials of Exondys 51 (eteplirsen), a recently approved analogous exon-skipping therapy for DMD marketed by Sarepta for patients with a confirmed mutation in the dystrophin gene that is amenable to skipping exon 51 . There was controversy surrounding the Exondys 51 approval by the FDA owing to the uncertainty of the functional benefit linked to small increases in dystrophin levels, and a major factor in its approval was the public pressure arising from patient families and advocacy groups, as an FDA advisory committee had voted slightly against approval of Exondys 51. Nonetheless, the FDA seems to recognize potential in Sarepta's novel approach to the treatment of DMD, as it recently indicated in a type C meeting that small increases in dystrophin production from baseline can form the basis of accelerated approval. Placebo-controlled efficacy data for golodirsen are unlikely to be available until the release of results from the ongoing phase III ESSENCE study, the primary endpoint of which is the 6-minute walk test. Sarepta has confirmed with the FDA that the ESSENCE trial can potentially serve as a post-marketing confirmatory study, should the treatment be approved. The PDUFA action date for golodirsen is 19 August.

Brandon Leavins

Sagient Research Systems, San Diego, CA, USA e-mail: Brandon.Leavins@informa.com https://doi.org/10.1038/d41573-019-00100-0

\section{Competing interests}

The author declares no competing interests. 